Article

\title{
Pinch Grasp and Suction for Delicate Object Manipulations Using Modular Anthropomorphic Robotic Gripper with Soft Layer Enhancements
}

\author{
Godwin Ponraj Joseph Vedhagiri ${ }^{1}$, Avataram Venkatavaradan Prituja ${ }^{1}$, Changsheng Li ${ }^{1}$, \\ Guoniu Zhu ${ }^{1}$, Nitish V. Thakor ${ }^{1,2}$ and Hongliang Ren ${ }^{1, *}$ D \\ 1 Department of Biomedical Engineering, National University of Singapore, Singapore 117575, Singapore \\ 2 Department of Biomedical Engineering, Electrical and Computer Engineering, and Neurology, \\ Johns Hopkins University, Baltimore, MD 21218, USA \\ * Correspondence: ren@nus.edu.sg or hlren@ieee.org
}

Received: 18 June 2019; Accepted: 3 August 2019; Published: 6 August 2019

check for updates

\begin{abstract}
This paper is an extension of our previous work about a modular anthropomorphic robotic hand with soft enhancements focusing on simultaneous pinch grasp and suction-based object manipulations. The base structure is a tendon-driven robotic hand comprising five fingers and a palm. Each finger consists of two rigid links covered with soft enhancements. The soft enhancements are like the skin and tissues of the robotic hand. The tip of the finger is equipped with a suction module which can be actuated by regulating negative pressure to the soft layers. While our previous work dealt with the rationale behind and the structure of the modular design with kinematic analysis, this paper focuses on analyzing two specific capabilities of the gripper-pinch grasp and suction modality. Experiments validate that the proposed gripper together with the soft enhancement layers is capable of performing delicate single finger suction-based manipulation tasks and two-finger pinch grasp tasks.
\end{abstract}

Keywords: soft robot applications; anthropomorphic robotic hand; contact modulation

\section{Introduction}

Robotic grippers have significantly influenced and revolutionized the workflow in several industries over the past few decades. With their high-speed operation, precise controllability and reproducibility, they increase the productivity of the manufacturing process. Now, robotic grippers have entered a more unconstrained dynamic environment and are soon to become an integral part of the day-to-day life of an average human being. The human hand, equipped with multiple somatosensory receptors under the epidermis and with the soft tissue structures covering the rigid skeleton, is able to handle a wide range of objects varying in size, shape, texture, weight, etc. Hence, a human hand is often considered as a model for creating an anthropomorphic robotic gripper. However, owing to the complexity in kinematics, sensing, and control, even the most sophisticated of the robotic grippers [1-3] are not able to achieve the dexterity and adaptability of a human hand.

A major area to improve is to bridge this gap between expectation and reality in the compliance of the grippers. It will allow the grippers to adapt to the object being handled and to better conform to their structure. Conventional robotic grippers have rigid links and form claw-like or hand-like structures to perform object handling tasks. A robotic hand with rigid bodies may be structurally stable. But rigid bodies possess the risk of exerting more excessive force on the object than needed. If the contact force is not monitored and left uncontrolled, the object being handled may get damaged.

Since the introduction of the domain of soft robotics, several grippers have been introduced that are entirely soft and have higher compliance [4]. However, they also have certain limitations. 
Complex control is required to achieve precise manipulation due to uncertain kinematic relations [5]. Soft structures might also not be able to provide the necessary grasping force to handle large objects [6] due to the lack of rigid support. It is also extremely difficult to sustain a particular body configuration in a dynamic environment.

These drawbacks could be overcome by providing a rigid framework to support the soft structures just like bones in a human body. It is also proven that under-actuated grippers with redundant degrees of freedom (DOFs) are better compliant than conventionally controlled grippers $[7,8]$. Developing soft robotic grippers with better compliance to improve dexterity or to develop existing grasp configurations has gained interest in the research community recently $[9,10]$. When a rigid robotic gripper handles an object, the interaction is often confined to the individual points where the robot contacts the object. However, soft structures can provide more contact area than their conventional counterparts due to their continuous flexibility and stretchability [4].

By carefully designing the shape and structure of these soft structures, various modalities like suction can be achieved even when handling small and irregularly shaped objects [11]. Even the stiffness of the soft structures can be varied by controlled actuation [12]. Employing suction to a gripper will improve the grasping capabilities and is employed in several industrial object handling end effectors [13,14]. This technology is inspired by nature from certain cephalopods that have suction based specialized anatomical features for object manipulation [15]. They are widely popular in industries that handles delicate objects like glass, plastic parts, food, etc., since they establish a soft contact with the object [16]. The suction cups attach to the surface of the object to create an air-tight boundary. When negative pressure is applied essentially creating a vacuum between the cup and the object, it holds on to the surface [17]. Even objects heavier than the suction cup itself with a non-porous surface can be lifted with sufficient negative pressure input. Research works have been done to improve such suction grasps focusing on the better suction interface [14], suction mechanism [18], control methodologies [19], etc.

However, there are very few research works that involve the combination of a rigid hand with a suction mechanism. An example of commercialized gripper that uses this hybrid combination is the Righthand Robotics [20]. This gripper has three flexible fingers arranged around a central extendable finger with suction cup to grasp objects. Due to the suction cup, the gripper can handle a wide range of delicate and irregular shaped objects without damaging them. In the Amazon Robotics Challenge, the teams that won the first prize in 2017 and 2015 took up this hybrid approach [21,22]. These teams had a rigid parallel-jaw gripper with an additional suction cup. The parallel jaw gripper is complementary to the suction cups. It is used for picking semi-porous materials that prevents a good suction seal whereas suction can be used to handle flat, larger objects.

Table 1 shows the consolidated information on some of the above-mentioned grippers or end effectors with the proposed gripper (last two rows of the table). While some of these existing works incorporate suction cups in together with rigid mechanisms, they do not adjust to delicate object structures like an independent soft actuator [14,23]. Integrated suction cups in [17] and [22] were only able to handle objects with planar and rigid surfaces. Instead one can have a dedicated finger to include a deformable suction cup as in [20]. However, it introduces an additional control maneuver in the overall task to extend/retract the suction lever. The novelty in our gripper is that it has layers of deformable soft structures to execute the suction mechanism on top of the existing rigid framework. These structures have added modalities which can be separately actuated. It can deform according to the curvature of the object whether the suction mechanism is being operated independently (e.g., a balloon) or in conjuncture with other grasping configurations (e.g., a large cylindrical container [24] or an egg [25]). The soft layers also play a mediatory role in transferring any change in grasp strength smoothly from the gripper to the object thus preventing any damage to the object. 
Table 1. Comparison of the proposed gripper [rows five (our previous work) and six (this work)] with existing representative grippers based on the suction modality.

\begin{tabular}{cccccc}
\hline S. No & Name & Type & Suction Mechanism & Objects * & Grasp Strategy * \\
\hline 1 & $\begin{array}{c}\text { Origami inspired } \\
\text { reconfigurable } \\
\text { suction gripper [14] }\end{array}$ & Soft & Soft suction gripper. & $\begin{array}{c}\text { Rigid and soft } \\
\text { objects: various } \\
\text { geometry and sizes. }\end{array}$ & $\begin{array}{c}\text { Reconfigure gripper to match object } \\
\text { geometry and engage suction. }\end{array}$ \\
\hline 3 & $\begin{array}{c}\text { Robot Hand with } \\
\text { Suction } \\
\text { Mechanism } \\
\text { (iGRIPP 4) [17] }\end{array}$ & Rigid & $\begin{array}{c}\text { Suction cups fixed } \\
\text { at fingertips. }\end{array}$ & $\begin{array}{c}\text { Rigid objects: planar, } \\
\text { large to } \\
\text { small cuboids. }\end{array}$ & $\begin{array}{c}\text { Position suction cups on planar } \\
\text { surfaces of the objects and engage. }\end{array}$ \\
\hline RightPick 2 & Rigid & $\begin{array}{c}\text { Separate extendable } \\
\text { finger at the centre } \\
\text { with suction cup. }\end{array}$ & $\begin{array}{c}\text { Rigid and soft } \\
\text { objects: cylindrical, } \\
\text { cuboid and } \\
\text { tubular objects. }\end{array}$ & $\begin{array}{c}\text { Extend middle lever for the suction } \\
\text { cup to make contact with the object } \\
\text { and engage suction. }\end{array}$ \\
\hline 5 & $\begin{array}{c}\text { Custom designed } \\
\text { gripper for } \\
\text { Amazon picking } \\
\text { challenge [22] }\end{array}$ & Rigid & $\begin{array}{c}\text { Suction cup attached } \\
\text { to the dorsal side of } \\
\text { one of the fingers. }\end{array}$ & $\begin{array}{c}\text { Rigid and planar } \\
\text { surface objects. }\end{array}$ & $\begin{array}{c}\text { Position suction cups on planar } \\
\text { surfaces of the object and engage. }\end{array}$ \\
\hline $\begin{array}{c}\text { Modular } \\
\text { anthropomorphic } \\
\text { robotic gripper [24] }\end{array}$ & Hybrid & $\begin{array}{c}\text { Deformable soft } \\
\text { structures enclose } \\
\text { the rigid finger. }\end{array}$ & $\begin{array}{c}\text { Rigid and soft } \\
\text { objects: various } \\
\text { shapes and sizes. }\end{array}$ & $\begin{array}{c}\text { Performs power grasp and engages } \\
\text { suction simultaneously for } \\
\text { better grasping. }\end{array}$ \\
\hline 6 & [This work] & Hybrid & $\begin{array}{c}\text { Deformable soft } \\
\text { structures (with } \\
\text { suction module) } \\
\text { enclose the } \\
\text { rigid finger. }\end{array}$ & $\begin{array}{c}\text { Rigid and soft } \\
\text { objects: small, thin, } \\
\text { planar, curved or } \\
\text { irregular surface. }\end{array}$ & $\begin{array}{c}\text { (1) Soft suction module deforms to } \\
\text { surface curvature of the object on } \\
\text { contact and engages suction; } \\
\text { (2) Pinch grasp with dynamic grasp } \\
\text { strength changes. Soft layers transmit } \\
\text { the change to the object smoothly. }\end{array}$ \\
\hline
\end{tabular}

* Objects and Grasp strategies which does not involve the suction mechanism or soft structures are not given in the table.

While the overall grasping movement is controlled by the rigid gripper framework, the suction module will enhance or facilitate local contact with the object. The rationale behind and the structure of the modular design with kinematic analysis are discussed in our previous paper [24].

As an extension, this paper discusses the application of the developed anthropomorphic robotic gripper in a specific grasp orientation-the pinch grasp and the suction modality of the soft enhancement layers. According to the GRASP taxonomy [26], which classifies the known human grasp orientations based on finger and palm positions, applied force, etc., two types of pinch grasps are prevalent in humans depending on the application. A tip pinch is when the object is held between the thumb and one of the other (often index) fingertips. In a palmar pinch grasp, the object is held between the thumb and the entire distal segment rather than just the tips. Out of these two types, the palmar pinch is used more frequently than the other type [27] by humans. The paper is organized as follows. Section 2 briefly describes the system architecture and components. Section 3 explains the experimental setup and discusses some observations. In the last section, conclusions and future research directions are given.

\section{System Description}

The design of the proposed robotic gripper was inspired by human hand anatomy which includes anthropomorphic appearance, digits with multiple segments and soft cover. The robotic gripper comprised five individual fingers, each consisting of two modules (Figure 1a). The modules were detachable and replaceable easily to facilitate quick installation and repair when needed. It further facilitated the addition or removal of modules to handle very large or small objects relatively. Each finger (with two modules) provided two degree of freedoms (DOFs) for its flexion/extension (Figure 1b). Additionally, two more DOFs are provided by the foldable palm and thumb for its abduction/adduction (Figure 1a). 


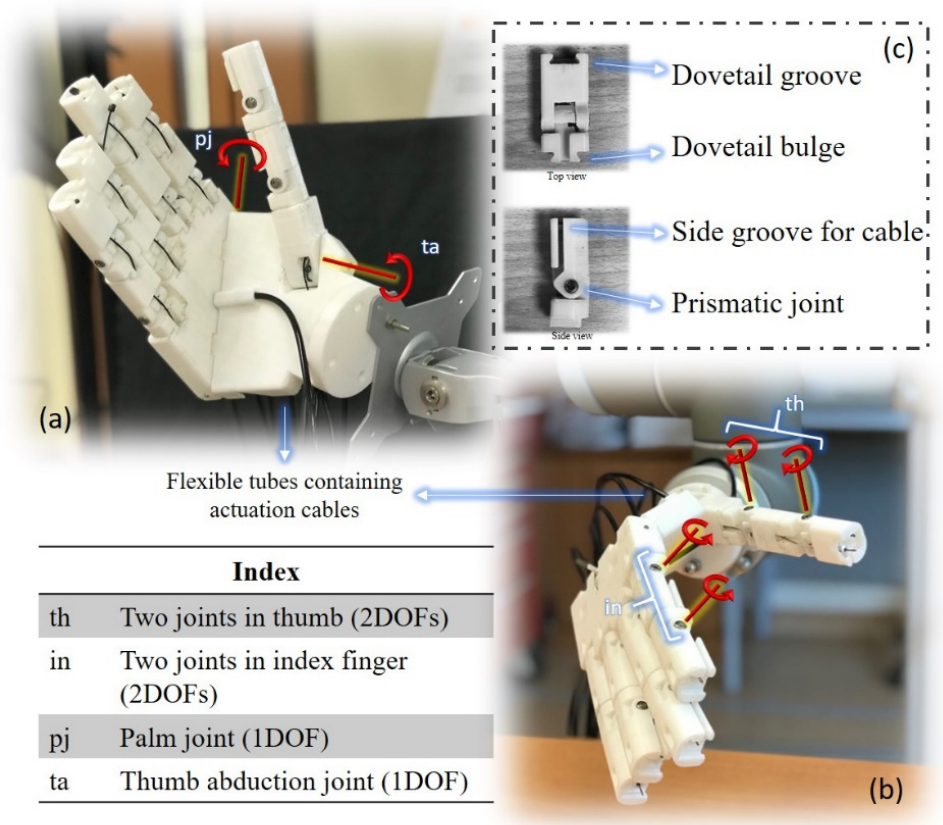

Figure 1. Modular anthropomorphic robotic gripper without soft enhancement layers. (a) View showing the palm joint and the thumb abduction joint each providing one degree of freedom (DOF). (b) View showing the two DOFs of thumb and index fingers. Similarly, two DOFs each will be provided by the other fingers. Arrows indicate the direction of rotation from open palm to closed fist. (c) Structure of a single module showing the grooves and bulge for module-to-module joint and actuation cable.

The foldable palm-joint can be considered as a fused MCP (Metacarpophalangeal) joint of the four fingers. It allowed the gripper to grasp objects of different sizes. The thumb abduction joint allows the thumb to come to a position to and facilitate the famous 'opposing thumb' functionality found in most primates including humans. Each of the fingers, the palm joint, and the thumb abduction joint were driven by seven servo motors via Bowden cables. The soft layer enhancements are analogous to the tissue and skin covering a human hand. They are accoutred to the rigid modules as functional enhancements. The fingers and the palm were fabricated out of polylactic acid (PLA), whereas the soft layers were made up of silicone.

\subsection{Modular Finger Structure}

Each finger of the gripper consisted of two modules. The dimensions of each module were $20 \mathrm{~mm} \times 25 \mathrm{~mm} \times 51 \mathrm{~mm}$. Each module also consisted of a prismatic joint to facilitate finger movement (Figure 1b). They were fabricated in a 3D printer (LulzBot TAZ 5, Aleph Objects, Inc., Loveland, CO, USA) using PLA. The connection between two adjacent modules and between the proximal module and the palm was via a dovetail joint. Each of the modules had a dovetail groove with spring plunger at the top end and a similarly shaped bulge at the bottom end (Figure 1c). For adding a module, the spring plunger was retracted to expose the groove at the top completely and the bulge of another module is slid in. When the spring plunger was released, the two modules were held together by its frictional engagement. This simple mechanism allowed easy adding or removing of the modules when needed.

Since the number of modules (two) was higher than the input control parameter (one servo motor), the proposed system became an under-actuated mechanism with higher compliance than full actuation system. Depending on the size of the object to be manipulated and precision requirement, the number of modules can be adjusted. For example, for a highly precise application requirement, single module fingers will provide better performance than two modules. Similarly, for a relatively large object, more than two modules might be needed to successfully manipulate it. 
The fingers are actuated by servo motors via a cable driven actuator mechanism. Each module has a staggered deep groove one on the side to allow the cable to run across to the next module (Figure 1c). Hence while adding or removing the modules, the cable running through them need not be altered. At the tip of the fingers, the cable is tied to a knot and a module cap with just the dovetail bulge is used to cover it. For better understanding, the modular design with kinematic analysis can refer to our previous work [9].

\subsection{Soft Enhancement Layers}

The soft enhancement layers were fabricated using EcoFlex ${ }^{\mathrm{TM}}$ 00-30 (Smooth-on, Inc., Macungie, PA, USA). The softness of this material is claimed to be closer to human skin and yet strong and stretchable. Two functionally distinct types of soft modules were designed and fabricated. The suction module, which operates on negative pressure, to cover the rigid links of the gripper and the inflation module, which operates on positive pressure, to cover the joints of the gripper.

The suction module consisted of four layers glued together to form a single module (Figure 2). They are (1) the base layer to act as a support and cover for the suction chamber; (2) the spacer layer to provide gap between the suction holes and the base layer, thus creating the suction chamber; (3) the suction layer with the suction holes; and (4) enclosure layer to provide airtight contact establishment with the object. The suction layer had four holes of diameter $5 \mathrm{~mm}$ each to maximize the suction effect [21]. The spacer layer had a small hole through which a soft tube of $1 \mathrm{~mm}$ diameter was inserted. A vacuum pump connected to the module via this soft tube will facilitate the actuation. The dimensions of the entire module were $20 \mathrm{~mm} \times 20 \mathrm{~mm} \times 6 \mathrm{~mm}$ approximately. When negative pressure was applied to the module, the air between the module and the object was sucked out via the holes in the suction layer creating a vacuum. Thus, the module was firmly attached to the object.
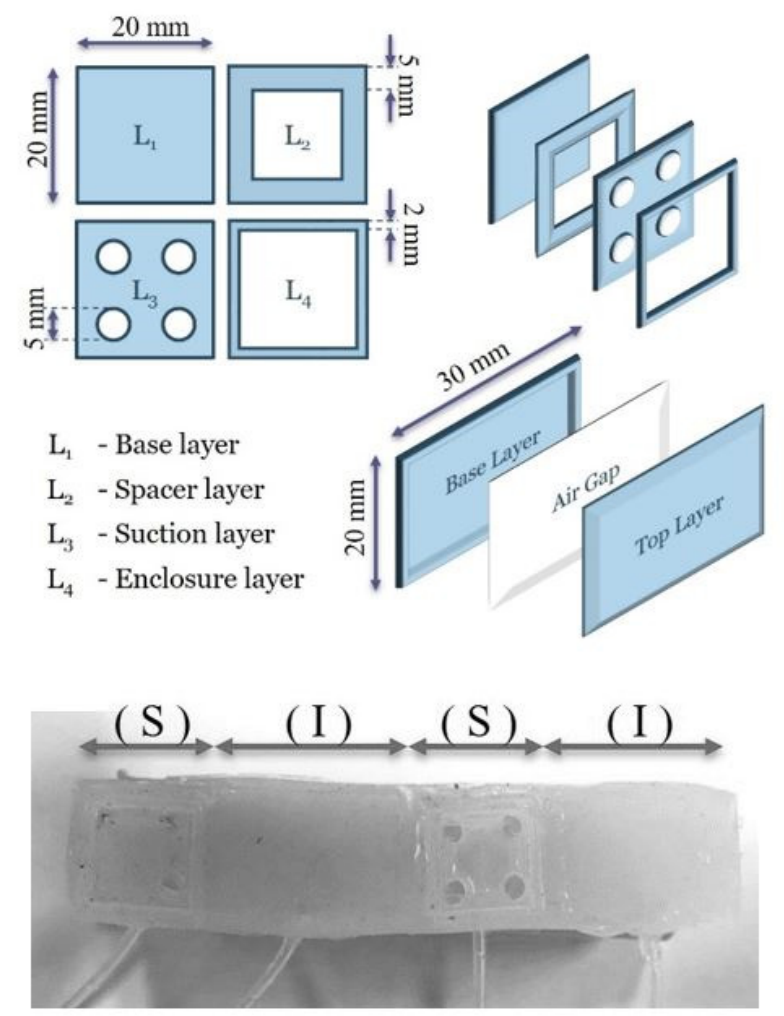

Figure 2. The layered architecture of the soft modules showing layer description of the suction module (top-left); orientation and arrangement of the layers in the suction module (top-right) and inflation module (middle); structure of the entire soft finger (bottom) with alternating suction $(S)$ and inflation (I) modules. 
The inflation module consisted of two layers of $2 \mathrm{~mm}$ thickness each. The layers were glued together at their boundaries except for a small hole. A soft tube was inserted in the hole and was sealed from outside forming an airtight chamber. A vacuum pump was connected to the soft tube to actuate the module similar to the suction module. However, the pump was operated to provide positive pressure instead, thus inflating the module. The dimensions of the inflation module were $20 \mathrm{~mm} \times 30 \mathrm{~mm} \times 4 \mathrm{~mm}$ (without actuation) approximately. The suction and inflation modules were glued together along their longest axis to form a single continuous structure. (Figure 2). Thin strips of silicone were glued to the distal and lateral ends of the suction module as extensions and loops to facilitate easy mounting over the modular fingers.

The vacuum pumps can be quickly installed with the soft tubes and can be kept separately, away from the working area without disturbing the gripper operation. While the overall grasping action and the orientation are controlled by the modular robotic gripper, the soft layers provide contact modulation to improve the grip. The soft enhancement layers, in general, can enhance friction for the modular fingers to grasp the objects firmly. It further provides simple protection against strong impact force for delicate objects by increasing the contact area and avoiding concentrated force. Additionally, a $4 \times 4$ multi-taxel flexible piezoresistive tactile sensor is also integrated along the back side of the base layer of the suction module. However, due to the scope of the paper, the tactile sensing and applications of the inflation module are not detailed here.

\subsection{Actuation and Control}

The modular gripper was actuated by Bowden cables where a thin cable of $1 \mathrm{~mm}$ diameter runs inside a flexible tube (outer diameter: $4 \mathrm{~mm}$, inner diameter: $2 \mathrm{~mm}$ ). This allowed the servo motors actuating the gripper to be situated away from the gripper itself. Each finger and the two additional palm and thumb abduction joints were driven by seven independent actuators which allowed full control in position, speed and torque modes. Dynamixel MX-64R servo motors (Robotis Inc, Seoul, Korea) were selected as the actuators due to their cascade communication capability and high torque to size ratio. Each of the seven servo motors was connected to a back-drivable tendon spool which could be passively rotated to adjust the length of the cable while adding or replacing the finger modules.

The servo motor actuators were controlled via two different control strategies using the joint torques and position values measured from their feedback signals as described below. The first control methodology was based on human hand gestures. It was used for position control of the gripper, where the fingertip positions were controlled to follow the human master. The input device used for this mode of control is the Leap Motion Controller (Leap Motion, Inc., San Francisco, CA, USA). It is a human hand motion tracking device comprising two monochromatic cameras and three infrared LEDs. The position, velocity and orientation information of the fingertips and palm of the human master hand was collected by this device and transmitted to the computer where a python-based program converted it into servo motor control parameters based on the kinematic model [9].

The second control strategy was based on the servo motor's torque. It was mainly used for controlling the grasping strength of the modular fingers. A required target torque could be specified as user input. A python script would drive the motors via a simple PI control loop until the feedback torque matched the target torque. The lower and upper torque value limits for the servo motors used were 0 and $6 \mathrm{Nm}$ respectively. The soft enhancement layers were actuated by an air pump (C183-22-01, Parker Hannifin, Singapore) with one negative-pressure channel (air pressure ranged from $-73 \mathrm{kPa}$ to 0 ) and one positive-pressure channel (air pressure ranged from 0 to $73 \mathrm{kPa}$ ) for the suction and inflation modules respectively. The control of the soft layers is manual wherein the suction or inflation pumps were actuated if and when necessary. The suction pump was always actuated with a negative pressure of $-73 \mathrm{kPa}$ whereas the positive pressure from the inflation pump varied depending on the space available for inflation.

The anthropomorphic gripper was attached as an end effector for a UR5 robotic arm (Universal Robots, Odense, Denmark). The robotic arm was controlled manually via its Polyscope Robot User 
Interface. Through the series of experiments done and as described in the following sections, it was used to position and move the robotic gripper in specific patterns to carry out the task.

\section{Experiments and Procedures}

\subsection{Pinch Grasp Experiments}

The developed anthropomorphic gripper is tested for a specific task in this section. Pinch grasp is a grasp orientation in which the object is held between the opposing thumb and the index and/or middle fingers. Humans use this orientation for objects whose thickness or diameter is normally smaller than the hand.

Two different objects of slightly varying thickness were used in the first experiment. However, the sequence of steps to be followed for the two objects changed slightly owing to their different sizes and different initial positions. The first object was a coin of $25 \mathrm{~mm}$ diameter, placed on top of a container (Figure 3a). The index finger was first used to move the coin towards the edge of the container (Figure 3b) such that a part of it was extending outside the container (Figure 3c). Then, the thumb was closed gradually as the hand was lifted slightly upwards (Figure 3d). This caused the coin to be trapped between the thumb and index fingers thus performing a tip pinch grasp (Figure 3e). The coin was later dropped inside another container as shown in Figure 3f. The experiment was repeated with the coin placed at random positions on top of the container. The gripper was able to pick up the coin 13 times out of the 17 trials done. Due to the small size and thickness of the coin, it was the object with least number of successful trials. Some failure cases included the coin being dropped down before the two fingers could hold it together (as shown in the video of Supplementary Materials).

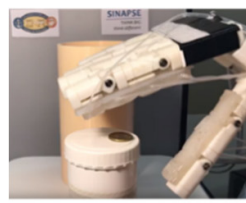

(a) $t=0: 00$

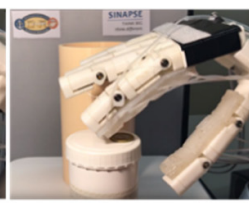

(b) $t=0: 03$

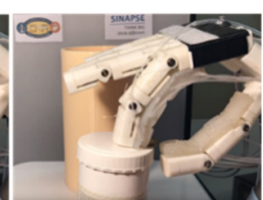

(c) $t=0: 04$

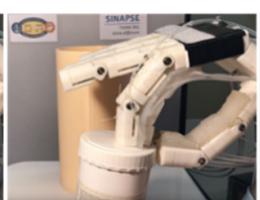

(d) $t=0: 06$

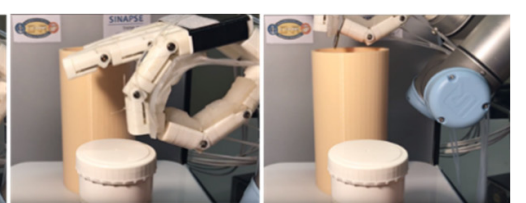

(e) $t=0: 08$

(f) $t=0: 24$

Figure 3. Modular anthropomorphic gripper picking up a coin in tip pinch grasp orientation. (a) Hand approaches the coin on top of a container. (b) Index finger makes contact with the coin. (c) Index finger slides the coin on to the edge of the container. (d) Thumb lifts up the coin slightly. (e) Coin picked up between the thumb and index finger in a tip pinch grasp. (f) The coin is dropped into a second container.

The second object was a toothpick of a diameter of $2 \mathrm{~mm}$ and length of $65 \mathrm{~mm}$. It was also placed on top of a container but with one-fourth of its length extending outside the boundary of the container (Figure 4a). Both the thumb and the index fingers were moved simultaneously (Figure $4 \mathrm{~b}$ ) and made contact with a toothpick around the same time (Figure 4c). As the index finger closed in, the gripper was moved outward and upward (Figure $4 \mathrm{~d}$ ). The toothpick then snapped between the two fingers in a quick motion (Figure 4e). Finally, the toothpick was returned to the top of the container as shown in Figure 4f. The experiment was repeated with the toothpick placed at various positions on the top of the container. However, the orientation of the toothpick with respect to the gripper was constant. The success rate of the toothpick experiment (14 out of 17) was slightly better than that of the coin owing to its length. In both of the experiments, torque-based control with a fixed target torque was used to actuate the fingers. However, certain object manipulation tasks might require the grasping strength of the fingers to vary dynamically during the task. 


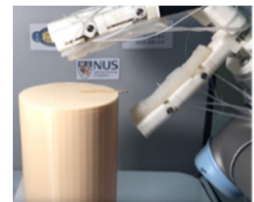

(a) $t=0: 00$

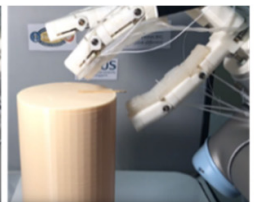

(b) $t=0: 02$

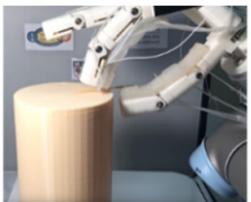

(c) $t=0: 03$

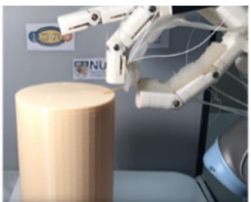

(d) $t=0: 04$

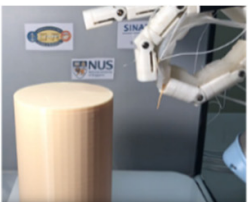

(e) $t=0: 08$

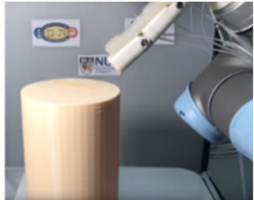

(f) $t=0: 29$

Figure 4. Modular anthropomorphic gripper picking up a toothpick in tip pinch grasp orientation. (a) Hand approaches the toothpick on top of the container with a quarter of its body extending outwards. (b) Both thumb and index fingers move toward the toothpick simultaneously. (c) Both the fingers make contact with the toothpick simultaneously. (d) The entire gripper is moved slightly outward and upward. (e) The toothpick is picked up between the thumb and the index finger in tip pinch grasp. (f) The toothpick is dropped back to the table.

Another set of experiments were done to explore such activities. To successfully achieve palmar tip pinch, the palm joint and the thumb abduction joints were actuated to bring the four fingers closer and almost parallel to the thumb. The objects used were a cosmetic cream tube and a lime fruit. The task was to grasp and squeeze to extract cream and juice from the two objects respectively. The cosmetics cream tube was placed on top of a small cube with the unscrewed lid facing down (Figure 5a). This was to facilitate easy opening of the tube by just lifting the body (Figure $5 b$ ). The tube was grasped along its length by the thumb and index fingers in a palmar pinch grasp orientation and was brought down such that the nozzle was near the tray (Figure $5 c$ ). The target torque was increased for the thumb which indirectly applied pressure on the tube so as to squeeze the cream out of it (Figure 5d).

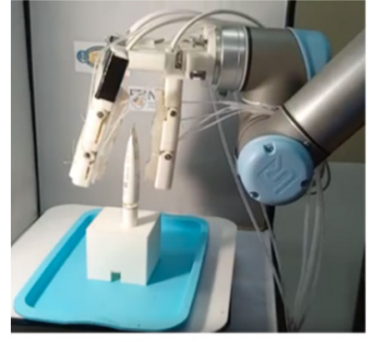

(a) $t=0: 01$

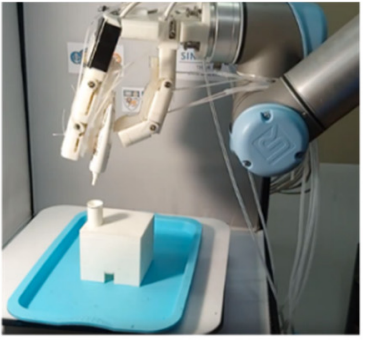

(b) $t=0: 06$

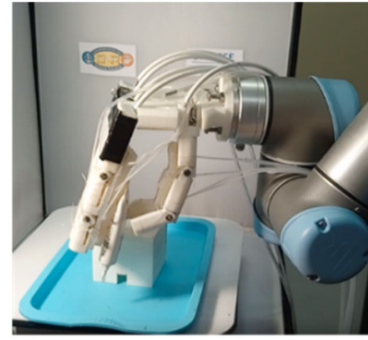

(c) $t=0: 17$

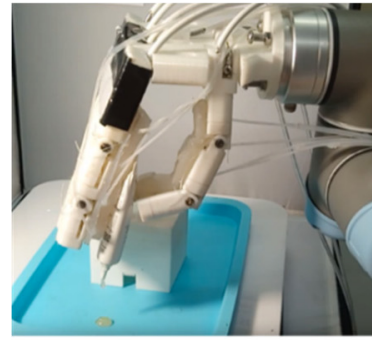

(d) $t=0: 41$

Figure 5. Modular anthropomorphic gripper manipulating a cosmetic cream tube. (a) Hand approaches the semi-opened cream tube. (b) The cream tube is picked up between the thumb and the index finger (c) and brought near the tray. (d) The cream is squeezed out of the tube by pressing the thumb against the index finger. It is achieved by increasing the target torque for the thumb while maintaining the index finger at the same position as previous.

The second object was a lime fruit with a portion of it sliced off and placed on the tray facing down (Figure 6a). It was picked up by the gripper using a palmar pinch grasp (Figure 6b). The robotic arm was moved to its right such that the gripper and the lime was now directly above a glass tumbler. Now, the target torque value was increased for the thumb which made it squeeze the lime against the index finger (Figure 6c). An enlarged view (Figure 6d) shows the juice being extracted and collecting in the glass. The experiments were repeated several times with different initial positions on the table. The success rates $(88.23 \%$ and $83.33 \%$ respectively) were higher than that of the coin and toothpick experiments. This is due to the fact that the objects used were bigger in size having higher contact area with the fingers. 


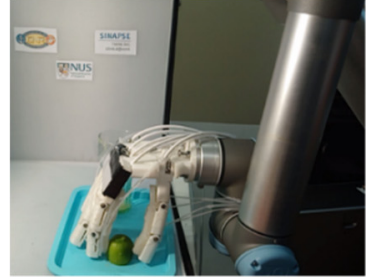

(a) $t=0: 01$

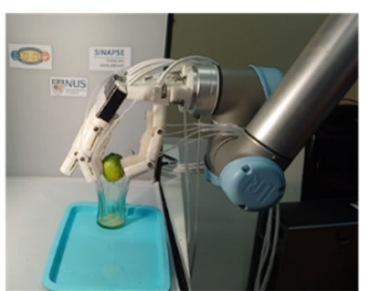

(b) $t=0: 12$

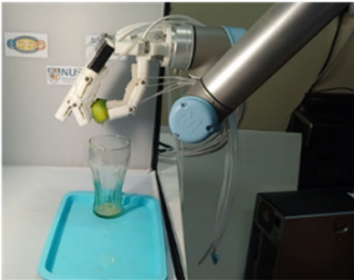

(c) $t=0: 26$

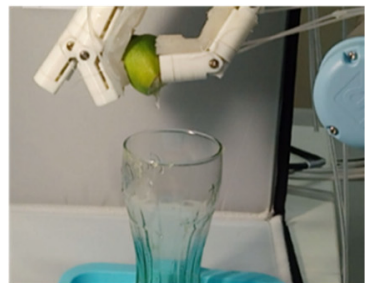

(d) $t=0: 31$

Figure 6. Modular anthropomorphic gripper manipulating a lime fruit. (a) Hand approaches the fruit. (b) The lime is picked up between the thumb and the index finger and brought over a glass tumbler. (c) The juice is squeezed out of the lime by pressing the thumb against the index finger. It is achieved by increasing the target torque for the thumb while maintaining the index finger at the same position as previous. (d) Enlarged view showing a juice drop from the lime.

The increase in grasp strength was transferred from the fingers to the objects via the soft enhancement layers in an efficient manner. Without the soft layers, a sudden change in contact force will often damage the object or result in it slipping out of the hand. It is interesting to note that the orientation of the fingers by the end of the tasks lies somewhere between the definition of a palmar pinch grasp and inferior pincer due to the relative motion of the thumb with respect to the index finger.

\subsection{Suction Experiments}

In the following set of experiments, the objects were handled using the suction module of the soft enhancement layers alone. This demonstrates the special ability of our design, unlike many other anthropomorphic grippers. In the first experiment, two objects of different size but similar weight were held by the hand. The first object was a medium sized balloon (diameter at the place of contact: $70 \mathrm{~mm}$ approximately)—a delicate object with soft, slippery and curved surface. Initially, the balloon was given to the hand by the operator, which then held it in a pinch grip between thumb and index fingers (Figure 7a). The suction module at the distal segment of the index finger was actuated with a negative pressure of $-37 \mathrm{kPa}$ (Figure $7 \mathrm{~b}$ ). As the thumb was now retracted back, the balloon was held in position by the index finger using suction alone (Figure 7c) without any grip from the rigid part of the hand. Finally, the negative pressure was released causing the balloon to fall off the finger (Figure 7d). The experiment was repeated several times. It was observed that the suction cup engagement was not successful for five out of the 17 trials. This could be attributed to the fact that the orientation of the curved surface of the balloon with respect to the index finger was not precisely controlled.

The second object was a credit card-a thin flat object with dimensions of $86 \mathrm{~mm} \times 54 \mathrm{~mm}$ and weighing $3.2 \mathrm{~g}$. The hand was spread open and kept facing down the object, such that the suction module at the index finger was directly above the centre of the card. It was slowly brought down till it touched the card and suction module was actuated with negative pressure (Figure 8a). The UR5 arm was moved up to lift the hand upwards. It can be seen in Figure $8 \mathrm{~b}$ that the card was attached to the index finger using the suction module. When the suction pressure was turned off, the card fell off to the table (Figure 8c). The experiment was highly repeatable with only one failure case out of the 17 trials. The suction pressure engaged varied between $-13 \mathrm{kPa}$ to $-15 \mathrm{kPa}$ during each iteration. High success rate of this experiment was due to the laminated surface of the card which made an ideal contact with the suction cup in the index finger.

Another experiment was conducted to showcase the suction capability of the hand in a sophisticated day-to-day activity. The hand was used to flip a page of a notebook using suction and wrist movements alone. Typically, a human hand flipping would involve several steps including, sliding a page across the rest to isolate it, lifting it up using pinch grip and finally turning it over to the other side of the book. Such an intuitive task is however complicated for an anthropomorphic hand. With the proposed suction capabilities, we have devised an alternate procedure for page flipping involving suction. 


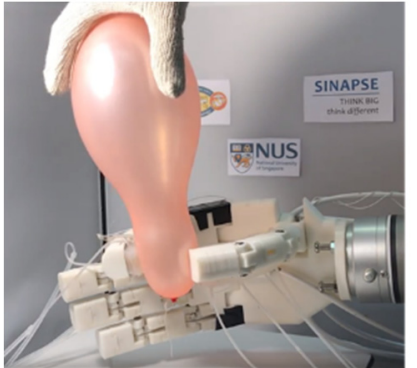

(a) $t=0: 01$

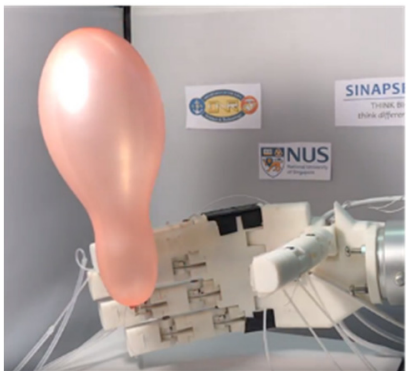

(c) $t=0: 09$

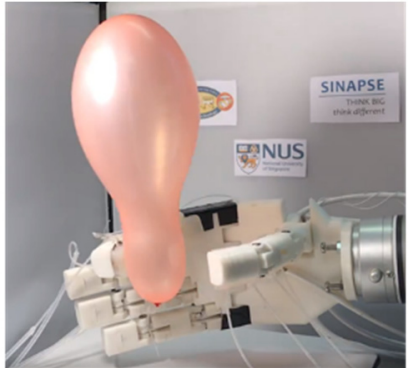

(b) $t=0: 08$

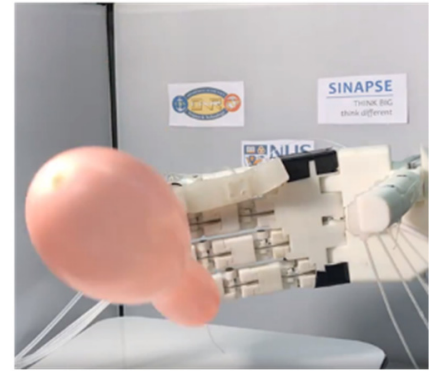

(d) $t=0: 29$

Figure 7. Modular anthropomorphic gripper manipulating a balloon with the suction module. (a) A full-blown balloon being given to the modular gripper by the operator. It is grasped between the thumb and index fingers. (b) The suction module at the tip of the index finger is engaged. (c) Negative pressure from the suction module holds the balloon even after the thumb is removed. (d) Balloon falls off the index finger after the suction pump is cut off.

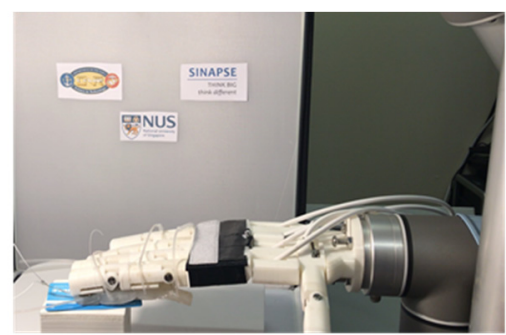

(a) $t=0: 00$

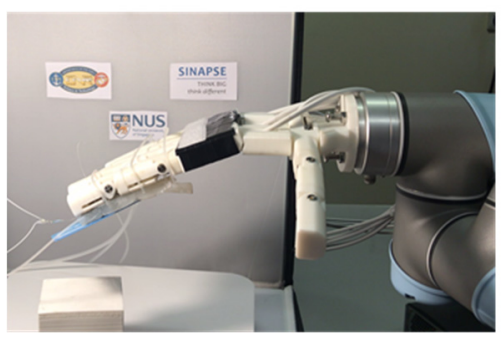

(b) $t=0: 07$

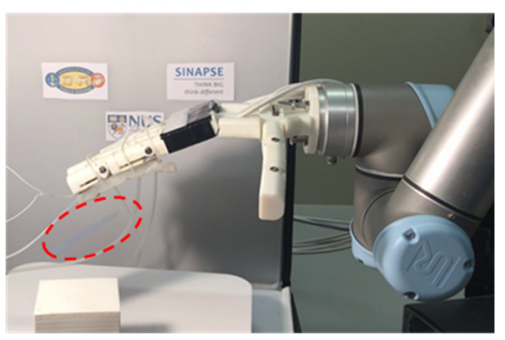

(c) $t=0: 13$

Figure 8. Modular anthropomorphic gripper lifting a credit card with the suction module. (a) Fully open hand approaches the card on top of a box and makes contact. (b) The suction module at the tip of the index finger is engaged. Negative pressure from the suction module holds the card even after it is lifted from the box. (c) The card falls off (dotted circle) the index finger after the suction pump is cut off.

The planar dimensions of the notebook used were $21.5 \mathrm{~cm} \times 14.5 \mathrm{~cm}$. The hand was spread open facing down such that the suction module was roughly positioned above the middle region of the left page margin ( 10-11 cm from the bottom). The hand was then pressed against the page along the edge and suction was engaged, (Figure 9a). The UR5 was used to slightly lift up the hand. It can be seen that the page was also lifted up slightly and was separated from the rest of the pages (Figure 9b). The wrist 
joint of the UR5 was then rotated clockwise (Figure 9c,d) gradually. The page followed the motion of the hand and began to turn. When the page crossed the centre of the notebook, the suction module was turned off (Figure 9e). This caused the page to dislodge from the finger. However, due to inertia, it continued the clockwise trajectory initiated by the hand (Figure $9 \mathrm{f}$ ). The hand was now slowly pulled out using the UR5 as it completed $180^{\circ}$ of clockwise rotation (Figure $9 \mathrm{~g}, \mathrm{~h}$ ). The page now falls against the right side of the notebook completing the flipping process. The suction pressure employed for this experiment was between $-23 \mathrm{kPa}$ to $-28 \mathrm{kPa}$. The average suction pressure for engaging a page was higher $(-25.6 \mathrm{kPa})$ than that of the card $(-13.9 \mathrm{kPa})$ because paper had comparatively higher porosity. It is to be noted that the notebook used in this experiment was a spiral bound notebook. This was to facilitate easy movement of the page when being flipped. The purpose of the experiment was to demonstrate that the proposed suction-based manipulation modality can be utilized for more than just pick and place tasks when properly combined with the gripper dynamics.

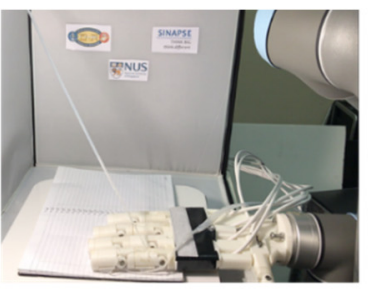

(a) $t=0: 00$

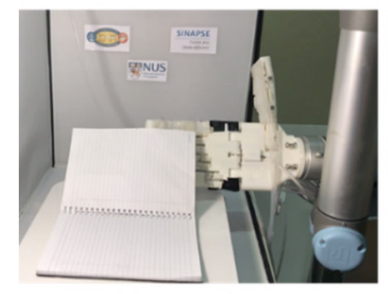

(e) $t=0: 10$

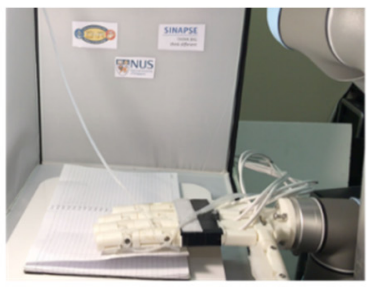

(b) $t=0: 07$

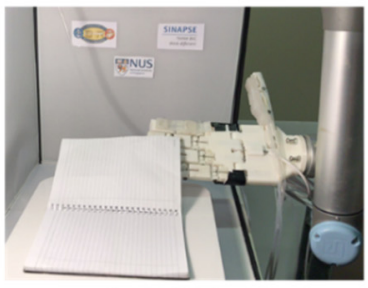

(f) $t=0: 10$

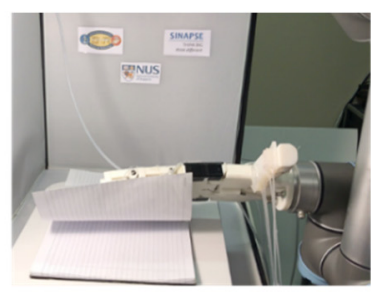

(c) $t=0: 08$

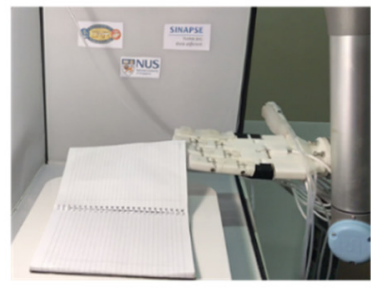

(g) $t=0: 11$

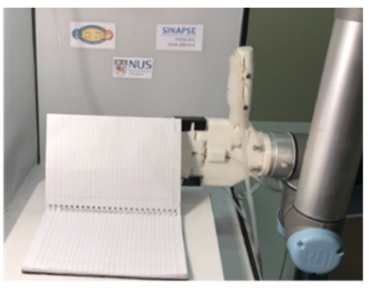

(d) $t=0: 09$

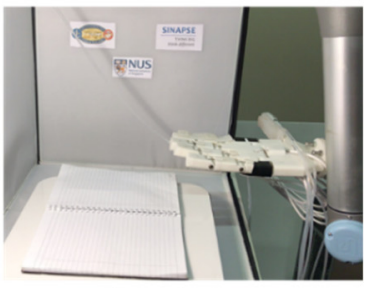

(h) $t=0: 12$

Figure 9. Modular anthropomorphic gripper manipulating a page with a suction module. (a) Fully open hand approaches the open notebook and makes contact such that the suction module is around the centre of the left margin of the left page. (b) The suction module is engaged, and the hand is lifted up slightly. (c,d) The wrist is rotated clockwise gradually. The page now moves along with the hand due to the negative pressure from the suction module. (e) Suction is cut off after the page has turned slightly more than half. (f-h) The hand is removed from under the page by slowly pulling out. The page continues the clockwise flip action due to inertia and completes a $180^{\circ}$ turn.

A major factor influencing the success of a suction mechanism is the ability to establish vacuum or create air-tight interface with the object. For the same negative suction pressure, the success rate varies for objects of different surface porosity. Higher porosity results in lesser success rate. However, by increasing the negative pressure, one can improve the success rate. Even though the paper is a planar object, it has minute pores on its surface which requires higher negative pressure to establish vacuum. In general, the suction modality will have higher success rate in planar surfaces without any surface irregularities. The success rate of all the experiments in terms of success percentage out of total number of trials is given in Table 2. 
Table 2. Success rates of the experiments.

\begin{tabular}{|c|c|c|c|}
\hline Ability & Experiment & Success Rate (\%) & Comments \\
\hline \multirow{4}{*}{ Pinch Grasp } & Coin & 76.47 & \multirow{2}{*}{$\begin{array}{l}\text { Objects to maintain same orientation with } \\
\text { respect to the gripper initially }\end{array}$} \\
\hline & Toothpick & 82.35 & \\
\hline & Cosmetic cream & 88.24 & \multirow{2}{*}{$\begin{array}{l}\text { Target torque increased for thumb } \\
\text { in between }\end{array}$} \\
\hline & Lime fruit & 83.33 & \\
\hline \multirow{3}{*}{ Suction } & Balloon & 70.59 & Single finger suction engaged \\
\hline & Card & 94.11 & Hand translational movement alone \\
\hline & Page flipping & 88.24 & $\begin{array}{l}\text { Hand twist and translation movements } \\
\text { with no individual finger movement }\end{array}$ \\
\hline
\end{tabular}

\section{Conclusions}

Pinch grasp and suction-based manipulation techniques using an anthropomorphic gripper with soft enhancements were discussed. The design and functionality of the different components of the gripper were first explained briefly. Easy, low-cost fabrication procedures and simple control strategies are the key advantages of the gripper. The experimentation sections show that the proposed gripper, in addition to simple object manipulation tasks, is also capable of performing certain complex tasks involving delicate and calculated finger movements.

With the presence of the soft enhancement layers, the fingers are able to transfer the force to the object gradually such that it is squeezed in a controlled manner. Similarly, the inclusion of suction modules in the anthropomorphic gripper has enhanced its capability and facilitates it to perform versatile manipulation modalities as explained in the previous section. As a follow-up, further work can be focused on analysing the application and utility of the suction modules on multiple fingers to explore more grasp orientations.

Supplementary Materials: A video compilation of all the experiments is available online at http://www.mdpi. com/2218-6581/8/3/67/s1, Video S1: Experiment_videos.

Author Contributions: Conceptualization, H.R. and G.P.J.V.; methodology, H.R., G.P.J.V., A.V.P. and G.Z.; software, G.Z.; data curation, G.P.J.V. and A.V.P; validation, G.P.J.V., C.L and A.V.P.; investigation, G.P.J.V.; resources, H.R.; visualization, G.P.J.V.; writing — original draft preparation, G.P.J.V.; writing—review and editing, H.R.; supervision, H.R.; project administration, H.R.; funding acquisition, H.R., and N.V.T."

Funding: This work is supported by the Office of Naval Research Global under grant ONRG-NICOP-N6290915-1-2029 and NUSRI China Jiangsu Provincial Grant BE2016077 awarded to Hongliang Ren.

Conflicts of Interest: The authors declare no conflict of interest.

\section{References}

1. Townsend, W. The BarrettHand Grasper-programmably flexible part handling and assembly. Ind. Robot. Int. J. 2000, 27, 181-188. [CrossRef]

2. Mouri, T.; Kawasaki, H.; Yoshikawa, K.; Takai, J.; Ito, S. Anthropomorphic robot hand: Gifu hand III. In Proceedings of the 2002 International Conference on Control, Automation and Systems (ICCAS), Jeonbuk, Korea, 16-19 October 2002; pp. 1288-1293.

3. Martin, J.; Grossard, M. Design of a fully modular and backdrivable dexterous hand. Int. J. Robot. Res. 2014, 33, 783-798. [CrossRef]

4. Rus, D.; Tolley, M.T. Design, fabrication and control of soft robots. Nature 2015, 521, 467-475. [CrossRef] [PubMed]

5. Tan, N.; Gu, X.; Ren, H. Design, characterization and applications of a novel soft actuator driven by flexible shafts. Mech. Mach. Theory 2018, 122, 197-218. [CrossRef]

6. Deimel, R.; Brock, O. Soft hands for reliable grasping strategies. In Soft Robot; Springer: Berlin/Heidelberg, Germany, 2015; pp. 211-221. 
7. Birglen, L.; Gosselin, C.M. Kinetostatic analysis of underactuated fingers. IEEE Trans. Robot. Autom. 2004, 20, 211-221. [CrossRef]

8. Li, C.; Gu, X.; Ren, H. A cable-driven flexible robotic grasper with lego-like modular and reconfigurable joints. IEEE/ASME Trans. Mechatron. 2017, 22, 2757-2767. [CrossRef]

9. Katzschmann, R.K.; Marchese, A.D.; Rus, D. Autonomous object manipulation using a soft planar grasping manipulator. Soft Robot. 2015, 2, 155-164. [CrossRef] [PubMed]

10. Al Abeach, L.A.; Nefti-Meziani, S.; Davis, S. Design of a variable stiffness soft dexterous gripper. Soft Robot. 2017, 4, 274-284. [CrossRef] [PubMed]

11. Liang, X.; Sun, Y.; Ren, H. A Flexible Fabrication Approach Toward the Shape Engineering of Microscale Soft Pneumatic Actuators. IEEE Robot. Autom. Lett. 2017, 2, 165-170. [CrossRef]

12. Huan, A.S.; Xu, W.; Ren, H. Investigation of a stiffness varying mechanism for flexible robotic system. In Proceedings of the 2016 IEEE International Conference on Mechatronics and Automation, Harbin, China, 7-10 August 2016; pp. 828-833.

13. Nakamoto, H.; Ohtake, M.; Komoda, K.; Sugahara, A.; Ogawa, A. A Gripper System for Robustly Picking Various Objects Placed Densely by Suction and Pinching. In Proceedings of the 2018 IEEE/RSJ International Conference on Intelligent Robots and Systems (IROS), Madrid, Spain, 1-5 October 2018; pp. 6093-6098.

14. Zhakypov, Z.; Heremans, F.; Billard, A.; Paik, J. An Origami-Inspired Reconfigurable Suction Gripper for Picking Objects with Variable Shape and Size. IEEE Robot. Autom. Lett. 2018, 3, 2894-2901. [CrossRef]

15. Walker, I.D. Continuum robot arms inspired by cephalopods" in et al. Int. Soc. Opt. Photonics 2005, 5804, 303-315.

16. Kumar, B. Vacuum gripper-an important material handling tool. Int. J. Sci. Technol. 2017, 7, 1-8.

17. Yamaguchi, K.; Hirata, Y.; Kosuge, K. Development of robot hand with suction mechanism for robust and dexterous grasping. In Proceedings of the 2013 IEEE/RSJ International Conference on Intelligent Robots and Systems, Tokyo, Japan, 3-7 November 2013; pp. 5500-5505.

18. Bamotra, A.; Walia, P.; Prituja, A.V.; Ren, H. Fabrication and Characterization of Novel Soft Compliant Robotic End-Effectors with Negative Pressure and Mechanical Advantages. In Proceedings of the 2018 3rd International Conference on Advanced Robotics and Mechatronics (ICARM), Singapore, 18-20 July 2018; pp. 369-374.

19. You, F.; Mende, M.; Štogl, D.; Hein, B.; Kröger, T. Model-Free Grasp Planning for Configurable Vacuum Grippers. In Proceedings of the 2018 IEEE/RSJ International Conference on Intelligent Robots and Systems (IROS), Madrid, Spain, 1-5 October 2018; pp. 4554-4561.

20. RightPick 2 Gripper. RightHand Robotics Inc. 2019. Available online: https://www.righthandrobotics.com/ (accessed on 25 July 2019).

21. Zeng, A.; Song, S.; Yu, T.K.; Donlon, E.; Hogan, R.F.; Bauza, M.; Fazeli, N. Robotic pick-and-place of novel objects in clutter with multi-affordance grasping and cross-domain image matching. In Proceedings of the 2018 IEEE International Conference on Robotics and Automation (ICRA), Brisbane, QLD, Australia, 21-25 May 2018; pp. 1-8.

22. Yu, T.K.; Fazeli, N.; Chavan-Dafle, N.; Taylor, O.; Donlon, E.; Lankenau, D.G.; Rodriguez, A. A summary of team mit's approach to the amazon picking challenge 2015. arXiv preprint arXiv:1604.03639, 2016.

23. Takahashi, T.; Suzuki, M.; Aoyagi, S. Octopus bioinspired vacuum gripper with micro bumps. In Proceedings of the 2016 IEEE 11th Annual International Conference on Nano/Micro Engineered and Molecular Systems (NEMS), Sendai, Japan, 17-20 April 2016; pp. 508-511.

24. Li, C.; Gu, X.; Xiao, X.; Zhu, G.; Prituja, A.V.; Ren, H. Transcend Anthropomorphic Robotic Grasping with Modular Antagonistic Mechanisms and Adhesive Soft Modulations. IEEE Robot. Autom. Lett. 2019, 4, 2463-2470. [CrossRef]

25. Ponraj, G.; Prituja, A.V.; Bamotra, A.; Guoniu, Z.; Kirthika, S.K.; Thakor, N.V.; Soares, A.B.; Ren, H. Active Contact Enhancements with Stretchable Soft Layers and Piezoresistive Tactile Array for Robotic Grippers. In Proceedings of the International Conference on Automation Science and Engineering (CASE), Vancouver, BC, Canada, 22-26 August 2019. 
26. Feix, T.; Romero, J.; Schmiedmayer, H.; Dollar, A.M.; Kragic, D. The GRASP Taxonomy of Human Grasp Types. IEEE Trans. Hum. -Mach. Syst. 2016, 46, 66-77. [CrossRef]

27. Keller, A.D.; Taylor, C.L.; Zahm, V. Studies to Determine the Functional Requirements for Hand and Arm Prosthesis; Dept. Eng. Los Angeles, Univ. California: Los Angeles, CA, USA, 1947.

(C) 2019 by the authors. Licensee MDPI, Basel, Switzerland. This article is an open access article distributed under the terms and conditions of the Creative Commons Attribution (CC BY) license (http://creativecommons.org/licenses/by/4.0/). 